\title{
Percepções de graduandos de nutrição sobre formação profissional em cuidados paliativos
}

\author{
Perceptions of nutrition undergraduates on professional education in palliative care \\ Percepciones de los estudiantes del grado en nutrición sobre la formación profesional en cuidados paliativos
}

\begin{abstract}
RESUMO
Objetivo: 0 presente estudo teve como objetivo identificar a abordagem de Cuidados Paliativos na formação acadêmica de discentes do curso de Nutrição de uma universidade federal do interior do Rio de Janeiro. Métodos: Trata-se de um estudo qualitativo, desenvolvido em 2018, com graduandos do Curso de Nutrição da Universidade Federal do Rio de Janeiro, Campus Macaé. Foi realizada uma exposição dialógica sobre o tema, seguida por aplicação de questionário semiestruturado e categorização temática das respostas. Resultados: Os discentes referiram que o tema Cuidados Paliativos foi pouco abordado na graduação e que seria fundamental para a formação ampliar esse conteúdo na matriz curricular. Conclusão: 0 presente estudo destaca fragilidades na formação em Cuidados Paliativos, que se correlacionam com lacunas gerais da formação em Nutrição e em saúde no Brasil. Reformulações curriculares são necessárias para aprofundar o conteúdo técnico na área e naturalizar discussões sobre a morte no ensino superior em saúde.
\end{abstract}

DESCRITORES: Cuidados Paliativos; Educação Superior; Capacitação de Recursos Humanos em Saúde.

\section{ABSTRACT}

Objective: This study aimed to identify the Palliative Care approach in the academic training of Nutrition undergraduates of a federal university in Rio de Janeiro. Methods: This is a qualitative study, carried out in 2018, with undergraduates from the Nutrition Program at the Federal University of Rio de Janeiro, Campus of Macaé. The participants attended a lectured on the subject, performed in a dialogical manner. They then answered a semi-structured questionnaire and it was done a thematic categorization of the answers. Results: The students reported that Palliative Care was hardly ever addressed during their courses and that expanding discussions on this topic was fundamental for their education. Conclusion: This present study sheds light on difficulties in Palliative Care education, which can be correlated to general gaps in nutrition and health training in Brazil. Curricular revisions are needed to deepen the technical content in the area and naturalize discussions about death in higher education. DESCRIPTORS: Hospitalized Child; Children's Rights; Nursing Education.

\section{RESUMEN}

Objetivo: Este estudio tuvo como objetivo identificar el enfoque de Cuidados Paliativos em la formación académica de estudiantes del curso de Nutrición de una universidade federal del interior de Río de Janeiro. Métodos: Se trata de una investigación cualitativa, desarrollada en 2018, con estudiantes del Grado en Nutrición en la Universidade Federal do Rio de Janeiro, Campus Macaé. Se realizó una exposición dialógica acerca del tema, seguida de la aplicación de una encuesta semiestructurada y categorización temático de las respuestas. Resultados: Los estudiantes informaron que son raras las veces en que la materia de Cuidados Paliativos es ministrada en la graduación y que sería fundamental para la formación ampliar este contenido en el plan de estudios. Conclusión: La investigación destaca las debilidades en la formación en Cuidados Paliativos, que se correlacionan con las brechas generales en la formación en nutrición y salud en Brasil. Se necesitan cambios curriculares para profundizar el contenido técnico em el área y naturalizar las discusiones sobre la muerte en la educación superior en salud. DESCRIPTORES: Cuidados Paliativos; Educación Superior; Capacitación de Recusos Humanos em Salud.

RECEBIDO EM: 15/06/2021 APROVADO EM: 20/07/2021 


\section{artigo}

Lacerda, M. G, Oliveira, R. B. A., Lourenço, A. E. P, Tavares, C. Q.

Percepções de graduandos de nutrição sobre formação profissional em cuidados paliativos

\section{MARIA GOUVÊA DE LACERDA}

Acadêmica de Medicina, UFRJ-Macaé. Nutricionista.

ORCID:0000-0002-0295-5471

\section{RENATA BORBA DE AMORIM OLIVEIRA}

Professora Adjunta do Curso de Nutrição, UFRJ-Macaé; Doutora em Ciências e Nutricionista. ORCID:0000-0001-6005-3460

\section{ANA ELIZA PORT LOURENÇO}

Professora Associada do Curso de Nutrição, UFRJ-Macaé; Doutora em Saúde Pública e Nutricionista. ORCIR:0000-0002-9619-8052

\section{CÁSSIA QUELHO TAVARES}

Professora Adjunta do Curso de Enfermagem e Obstetrícia, UFRJ-Macaé. Doutora em Teologia, Enfermeira e Teóloga. ORCID:0000-0002-9033-982X

\section{INTRODUÇÃO}

C uidados Paliativos (CP) são cuidados holísticos e ativos prestados à indivíduos com sofrimento intenso decorrente de doença, em especial àqueles que encontram-se próximos ao fim de vida. Têm como foco o paciente, e não sua enfermidade, com o objetivo de melhorar a qualidade de vida, incluindo a atenção com os familiares e cuidadores ${ }^{12}$. Por meio da Resolução no 41 de 31 de outubro de 2018, que dispōe sobre as diretrizes para CP no âmbito do Sistema Único de Saúde (SUS), o Brasil incorporou esta abordagem de cuidado no serviço de saúde ${ }^{3}$.

Trata-se então de uma incorporação recente e, por conseguinte, a formação e a atuação profissional em CP no Brasil ainda necessitam amadurecimento ${ }^{4}$. As habilidades que a prática de $\mathrm{CP}$ requer convergem com as diretrizes curriculares propostas para cursos na área da saúde, dentre eles o curso de Nutrição, que aborda a necessidade de uma formação humana, crítica e reflexiva ${ }^{5}$.

Um desafio basilar é que a matriz curricular nos cursos de graduação em Nutrição no país historicamente apresenta foco biomédico, com pouco espaço para o aprendizado em saúde coletiva e humanidades, que constituem alicerce aos graduandos e futuros profissionais para a prática da humanização e o desenvolvimento do pensamento crítico e da capacidade de lidar com situaçōes complexas ${ }^{6}$.

Graduaçōes implantadas mais recentemente no âmbito da expansão da educação superior no Brasil ${ }^{7}$ podem estar mais permeáveis a ajustes educacionais. O Curso de Nutrição da Universidade Federal do Rio de Janeiro - Campus Macaé Professor Aloísio Teixeira (UFRJ-Macaé), insere-se nessa conjuntura. Implantado em 2009, seu corpo docente se constituiu recentemente, as matrizes curriculares ainda estão em construção e desde o início, busca-se ultrapassar a dualidade entre a compreensão da saúde a partir de fenômenos biológicos em contraposição aos fenômenos sociais ${ }^{8}$.

Como forma de trazer elementos para subsidiar a implementação de conteúdos de $\mathrm{CP}$ na matriz curricular de cursos de graduação em Nutrição, o presente estudo teve como objetivo identificar a abordagem da temática na formação acadêmica de discentes do Curso de Nutrição da UFRJ-Macaée reafirmar a importância da atuação do profissional nutricionista na área de $\mathrm{CP}$.

\section{MÉTODOS}

Trata-se de uma pesquisa descritiva e exploratória, com abordagem qualitativa, desenvolvida em maio de 2018, com 15 estudantes do Curso de Nutrição da UFRJ-Macaé. Os critérios de inclusão para realização desse estudo foram: graduandos regularmente matriculados no $7^{\circ}$ período do Curso de Nutrição da referida universidade, de ambos os sexos e que concordaram em participar da pesquisa através da assinatura do Termo de Consentimento Livre e Esclarecido (TCLE). Os critérios de exclusão foram: alunos de outros cursos, de perí- odos anteriores ou posteriores ao preconizado, aqueles que se recusaram a participar ou não assinaram o TCLE.

Com o objetivo de identificar as percepçōes dos futuros nutricionistas, foi feita uma exposição dialogada sobre a temática, caracterizada por um encontro com duração aproximada de 6 horas divididas em turno da manhã e tarde, tendo como facilitadoras uma docente e uma discente do último período do curso de Nutrição da UFRJ-Macaé. Foram propostas reflexōes sobre finitude a partir da exibição de vídeos e da discussão em grupo sobre conceitos, princípios e diretrizes de CP.

A exposição dialogada seguiu o modelo educativo dialógico proposto por Paulo Freire' ${ }^{9}$ pautado na ausência de hierarquia de saberes, buscando valorizar a reflexão coletiva, a participação ativa dos graduandos, a interação de conhecimentos entre todos os atores e a reciclagem de saberes. Essa abordagem tem capacidade de levar todos os envolvidos ao questionamento, $\mathrm{e}$ formar pessoas mais reflexivas a fim de estimular a transformação da realidade.

Ao final, com vistas a identificar as percepçōes dos estudantes sobre o tema, os participantes responderam por escrito, de forma auto aplicada e individual, um questionário semiestruturado, desenvolvido especificamente para fins deste estudo, contendo perguntas abertas, cujos eixos centrais eram: a abordagem da temática na formação acadêmica e a importância da atuação do profissional nutricionista na área de CP. 
A análise do conteúdo qualitativo ocorreu por categorização temática ${ }^{10},{ }^{11}$. Os temas foram inicialmente identificados durante uma primeira leitura do material, explorados e agrupados de acordo com sua semelhança, e posteriormente interpretados e organizados em categorias, confrontando o material coletado com a literatura disponível do tema.

O planejamento, execução e interpretação dos resultados teve duração total de 12 meses. Toda execução do trabalho se baseou na Resolução n ${ }^{\circ} 510 / 2016$, que discorre sobre a ética, sigilo e respeito à dignidade em pesquisas envolvendo seres humanos. A pesquisa foi aprovada pelo Comitê de Ética em Pesquisa em Humanos da UFRJ-Macaé (registro $\mathrm{n}^{\circ}$ 79452417.5.0000.5699) e todos os participantes foram previamente esclarecidos sobre os procedimentos do estudo e assinaram o TCLE.

\section{RESULTADOS}

O conteúdo das respostas dos participantes foi analisado na íntegra. Para identificar os aspectos que foram objeto de investigação do presente estudo, foram definidas categorias temáticas por núcleos de sentidos dos discursos, conforme descrito abaixo:

\section{A abordagem da temática na formação acadêmica}

Sobre a abordagem do tema CP na formação profissional de futuros nutricionistas, os participantes destacaram que tinham pouca aproximação com o assunto. Um aluno comentou que "Não tinha noção sobre o tema e muito menos ouvi sobre na graduação" (A). Em unanimidade, os participantes responderam ser necessário ampliar a discussão sobre CP na graduação, a fim de favorecer a formação de profissionais mais preparados e críticos: "Acho que $[\mathrm{CP}]$ é algo que deve ser discutido com mais profundidade [na graduação] não apenas para nutrição, mas para todos os cursos da área da saúde" (B).

A atuação do profissional nutricionista na área de CP

\section{É priorizada a aprendizagem}

dos cuidados

terapêuticos com

ênfase curativa, $e$

não se prepara os

futuros profissionais

para lidarem a

morte e o morrer
Os discentes destacaram a importância do nutricionista na área de CP, comentando que "o alimento é algo que permeia nossa vida e pode contribuir de forma positiva [nos CP]" (C). Foi ressaltada também a possibilidade de protagonismo profissional nessa área, no contexto de trabalho em equipe multidisciplinar: "Considerando a alimentação como chave para o bem-estar, me sinto um grande influenciador da equipe para melhorar o atendimento ao paciente próximo ao fim da vida” (D). Porém alguns registros revelaram entraves relacionados à atuação em equipe e a autonomia profissional do nutricionista: "Às vezes nos sentimos um coadjuvante na equipe de saúde" (E).

\section{DISCUSSÃO}

Os participantes percebem o tema CP como relevante para sua formação profissional, porém destacam que pouco foram expostos ao assunto, o que pode contribuir para que se sintam despreparados para atuar na área. Esses desafios profissionais em lidar com pacientes em CP podem estar correlacionados com lacunas de formação e poucas discussões teóricas referentes à finitude humana ${ }^{4}$.

É priorizada a aprendizagem dos cuidados terapêuticos com ênfase curativa, e não se prepara os futuros profissionais para lidarem a morte e o morrer. Percepção semelhante foi observada em pesquisas anteriores, que apontaram que alunos, residentes e profissionais de diferentes áreas da saúde (incluindo Enfermagem, Medicina e Terapia Ocupacional), não se sentiam preparados para lidar com a morte pela ausência destas discussões na graduação e aprendiam sobre o assunto apenas quando vivenciavam situações de morte e morrer em suas vidas pessoais ou profissionais ${ }^{12}, 13$.

Os participantes enfatizaram a importância de ampliar a discussão sobre CP na graduação, salientando a potencialidade da temática para desconstruir percepções estritamente biologistas e agregar criticidade e humanização à formação. Essa colocação alinha-se com o que recomendam as diretrizes curriculares para os cursos de Nutri- 


\section{artigo}

Lacerda, M. G, Oliveira, R. B. A., Lourenço, A. E. P, Tavares, C. Q.

Percepções de graduandos de nutrição sobre formação profissional em cuidados paliativos

ção ${ }^{5}$ e traz à tona a discussão sobre a formação em saúde no Brasil. Humanizaçāo e criticidade são nós críticos dessa discussão. Nos cursos de Nutrição, disciplinas da área biomédica e tecnológica predominam nas matrizes curriculares, enquanto as de vertente social e humana representam uma menor fração da carga horária ${ }^{6}$.

Um caminho inicial para favorecer a formação em CP seria a criação de disciplinas optativas. Tais encontros não necessariamente alcançariam todos os estudantes matriculados, mas podem oferecer uma aproximação para aqueles que os vivenciam, ampliando a formaçāo teórico-prática na área. Além disso, disciplinas optativas costumam ser maleáveis no que se refere a integrar docentes e discentes de diferentes cursos, o que favoreceria o caráter interdisciplinar e interprofissional que se espera na atuação em $\mathrm{CP}^{1}$.

Os respondentes do presente estudo mencionaram desafios relacionados a atua- ção de nutricionistas em equipes de saúde, sendo citada a sensação de impotência, papel coadjuvante e falta de envolvimento entre a equipe. Tais dificuldades podem estar relacionadas com a identidade profissional do nutricionista, que ainda se encontra em construção e envolve um processo de busca por autonomia profissional ${ }^{14}$.

Um outro estudo também concluiu que enfermeiros de uma equipe multiprofissional de CP enfrentam dificuldade de realizar tais práticas devido à falta de formação e pouca discussão acerca da temática nos espaços hospitalares, com outros membros da equipe ${ }^{15}$. Especificamente em CP, é primordial a atuação conjunta e harmônica de diferentes profissionais, de maneira a permitir que os integrantes se comuniquem e que cada um possa contribuir efetivamente, dentro de sua área de atuação, para o cuidado integral dos pacientes ${ }^{16}$. Por se tratar de uma abordagem acolhedora para nutricionistas, pode consistir num campo de prota- gonismo dos profissionais.

\section{CONCLUSÕES}

Foi possível constatar fragilidades na formação em CP dos discentes investigados, que se correlacionam com lacunas gerais da formação em Nutrição e em saúde no Brasil. Sendo assim, sugere-se pensar em reformulações curriculares, principalmente no sentido de agregar à formação biomédica aprofundamentos acerca da humanização dos processos de trabalho e da atuação em equipe multiprofissional, além da incorporação de reflexões e conteúdos específicos sobre CP.

Espera-se aprofundar e naturalizar as discussões sobre terminalidade da vida no ensino superior em saúde no país, devendo-se buscar o aprimoramento para a atuação profissional em CP, favorecendo o potencial humano de cada um no cuidado ao outro que sofre.

\section{REFERÊNCIAS}

1. Academia Nacional de Cuidados Paliativos. Manual de Cuidados Paliativos: ampliado e atualizado. $2^{\text {a }}$ ed. Rio Grande do Sul: Solo: editoração e design gráfico; 2012

2. Radbruch L, Lima L, Knaul F, Wenk R, Ali Z, Bhatnaghar S, et al. Redefining Palliative Care - a New Consensus-based Definition. Journal of Pain and Symptom Management. 2020;60(4):754-64. https:/ doi. org/10.1016/j.jpainsymman.2020.04.027

3. Brasil. Ministério da Saúde. Gabinete do Ministro. Comissão Inter gestores Tripartite. Resolução $n^{\circ}$. 41, de 31 de outubro de 2018. As diretrizes para a organização dos cuidados paliativos, à luz dos cuidados continuados integrados, no âmbito Sistema Único de Saúde (SUS). Diário Oficial da União. Brasília, 23 de novembro de 2018; Seção 1, p.276

4. Faillace GBD. 0 ensino de cuidados paliativos na formação do nutricionista. Demetra: Alimentação, Nutrição \& Saúde. 2015;10(1):133-40. https://doi.org/10.12957/demetra.2015.13183

5. Brasil. Resolução $n^{\circ}$ 5/CNE/CES. Institui as Diretrizes Curriculares Nacionais do Curso de Graduação em Nutrição. Diário Oficial da União 7 nov. 2001.

6. Alvez CGL, Martinez MR. Lacunas entre a formação do nutricionista e o perfil de competências para atuação no Sistema Único de Saúde (SUS). Interface - Comunicação, Saúde, Educação. 2016;20(56):15969. https://doi.org/10.1590/1807-57622014.1336

7. Brasil. Ministério da Educação. Secretaria de Educação Superior (SESu). A democratização e expansão da educação superior no país 2003-2014. Brasilia: SESu; 2015

8. Lourenço AEP, Cordeiro, AA, Capelli, JCS, Oliveira RBAO, Pontes PV, Almeida MFL, et al. Programa de Educação pelo Trabalho para a Saúde (PET-Saúde) e a formação do nutricionista num campus de interior- ização. Demetra: Alimentação, Nutrição \& Saúde. 2017;12(1):41-58. https://doi.org/10.12957/demetra.2017.26214

9. Freire P. Pedagogia do oprimido. 46. ed. Rio de Janeiro: Paz e Terra; 2005

10. Vaismoradi $M$, Turunen $H$, Bondas $T$. Content analysis and thematic analysis: Implications for conducting a qualitative descriptive study. Nursing and Health Sciences. 2013;15:398-405. https:/doi. org/10.1111/nhs. 12048

11. Bardin, L. Análise de conteúdo. São Paulo: edições 70, 2011.

12. Vieira TA, Oliveira M, Martins ERC, Costa CMA, Alves RN, Marta CB. Cuidado paliativo ao cliente oncológico: percepções do acadêmico de enfermagem. Revista Online de Pesquisa Cuidado é Fundamental. 2017;9(1):175-80. https://doi.org/10.9789/2175-5361.2017. v9i1.175-180

13. Sartori AV, Battistel ALHT. A abordagem da morte na formação de profissionais e acadêmicos da enfermagem, medicina e terapia ocupacional. Caderno Brasileiro de Terapia Ocupacional. 2017;25(3):497-08. https://doi.org/10.4322/2526-8910.ctoA00770

14. Banduk MLS, Ruiz-Moreno L, Batista NA. A construção da identidade profissional na graduação do nutricionista. Interface - Comunicação, Saúde, Educação. 2009;13(28):111-20. https://doi.org/10.1590/ S1414-32832009000100010

15. Passos FA, Fonseca DA, Olário PS, Pinto ACS, Moreira MC, Pestana LC, et al. A percepção do enfermeiro acerca da desospitalização em cuidados paliativos. Revista Saúde Coletiva (Barueri). 2019;09(51):1966-72.

16. Caldas GHO, Moreira SNT, Vilar MJ. Cuidados paliativos: uma proposta para o ensino da graduação em medicina. Revista Brasileira de Geriatria e Gerontologia. 2018;21(3):261-71. https://doi. org/10.1590/1981-22562018021.180008 